\title{
Underdetermining the truth
}

Peter Lipton

\begin{abstract}
Beyond Positivism and Relativism: Theory, Method and Evidence. By Larry Laudan. Westview: 1996. Pp. 277. $\$ 59.95$ ( $h b k$ ); $\$ 22.95$ (pbk).
\end{abstract}

FOR scientists, data often provide a daunting constraint on theory: the challenge is to find even one theory that fits all the relevant evidence. By contrast, philosophers, particularly those of a sceptical inclination, have been impressed by the weakness of the empirical constraint. One of the most powerful weapons in the sceptic's arsenal is the argument from empirical equivalence. The sceptic grants the truth of whatever we choose to count as our evidence, but denies us knowledge of the claims we infer from it, on the grounds that there will always be contrary claims that are also compatible with that evidence. Thus, in the seventeenth century, René Descartes famously argued that the evidence of the senses could not possibly yield any knowledge of the external world, because that evidence is always compatible with the contrary hypotheses that we are just dreaming or under the influence of a malign and powerful deceiver.

Arguments from empirical equivalence have also attracted philosophers of science in the present century. For example, Willard van Orman Quine, the distin-

\section{New in paperback}

Dance for Two by Alan Lightman.

Pantheon, \$12. A collection of essays examining the way literary and scientific concerns can be brought into harmony.

\section{The Ghost of the Executed Engineer:} Technology and the Fall of the Soviet Union by Loren R. Graham. Harvard University Press, $£ 7.95$. The life of the engineer Peter Palchinsky in Stalinist Russia.

\section{Children of Choice: Freedom and the New Reproductive Technologies by John A. Robertson. Princeton University Press, \$16.95, £13.95. Examines the repercussions of developments in reproductive techniques.}

Physical Origins of Time Asymmetry edited by J. J. Halliwell, J. PérezMercader and W. H. Zurek. Cambridge University Press, £24.95, \$39.95.

Universes by John Leslie. Routledge, $£ 10.95$. A philosophical look at the question 'Why does our Universe exist?'

Chemistry Imagined: Reflections on Science by Roald Hoffmann and Vivian Torrence. Smithsonian Institution Press, £15.50. guished philosopher and logician, has claimed that all scientific theories are 'underdetermined' by the evidence. However much data a theory may have in its favour, there will always be in principle innumerable other theories, inconsistent with the first but consistent with these data. The underdetermination thesis has opened the door to philosophical accounts that deny realism, the view that science is capable of generating increasingly accurate representations of a largely unobservable world. According to some philosophers, the moral of underdetermination is that scientific theories can only be calculation devices for generating predictions; according to others, the slack that the data leave must be taken up by varying social and political factors, so the only defensible view of science is wholesale relativism.

Several of the essays in this stimulating collection by Larry Laudan, a prolific and influential philosopher of science, aim to undermine underdetermination. Laudan argues that the underdetermination thesis is ambiguous, and that the acceptable version is also epistemically innocuous. All that version shows is that theories can never be deduced from the data, which ought to be a truism. The support that evidence provides for theory will never amount to a proof. A threatening version of the underdetermination thesis would have to claim that, however extensive the evidence, there will always be innumerable competing theories that would be equally well supported by that evidence, a position that Laudan claims is implausible, and undefended by the friends of underdetermination. Laudan also argues that the notion of empirical equivalence is ill-defined, because the class of possible evidence that could bear on a theory varies over time. The auxiliary statements that may be used in concert with a theory to generate testable predictions will change; so will the class of observable states of the world, with innovations in instrumental technology. In this way, two old theories that could not be evidentially distinguished on the basis of naked-eye observation may well have become distinguishable with the invention of the microscope.

Given Laudan's robust criticism of underdetermination, one might expect him to offer a realist account of science. As other chapters in his book show, however, this is very far from the truth. He denies that scientific development is cumulative: new theories do not in general explain everything that their dethroned predecessors explained. For example, Descartes's vortex theory of planetary motion explained why all the planets move in the same direction, whereas Newton's theory did not. He also claims that both the aims and methods of science vary within and between scientific communities. Finally, although various different and incompatible scientific aims are legitimate, the realist's aim of discovering the truth about the world is not.

Laudan scorns relativism, which he regards as the occupational disease of recent philosophy of science, with Thomas Kuhn cast as a particularly virulent influence. Given Laudan's own position, however, one could be forgiven for thinking that he has himself been infected. Laudan nevertheless maintains that his own account is immune to relativism. Legitimate aims and methods may vary, and truth is not on the cards; but it is supposed to be an objective matter whether a particular method is a reliable means for realizing a particular aim. Laudan claims that the effectiveness of a scientific method is itself susceptible to empirical investigation, by testing for the correlation between the application of the method and the realization of the aim, in particular episodes in the history of science. Moreover, although Laudan firmly rejects a cumulative view of the development of science, he holds that science is objectively progressive, because later theories solve more problems than their predecessors, even though some old solutions are dropped without replacement.

Laudan's pluralistic account of science is genuinely different from simple relativism, but his presentation is open to challenge. Although their positions are not the same, Laudan misrepresents Kuhn in a way that exaggerates the contrast. In his classic Structure of Scientific Revolutions (University of Chicago Press, 2nd edn, 1970), Kuhn writes: "Later scientific theories are better than earlier ones for solving puzzles in the often quite different environments to which they are applied. That is not a relativist's position, and it displays the sense in which I am a convinced believer in scientific progress."

Laudan is also at one with Kuhn in the rejection of the realist view of truth as a legitimate aim of science. Although the issues are complex and Laudan has elsewhere provided direct arguments against realism, his criteria for legitimate aims may beg the question against truth. $\mathrm{He}$ holds that a pairing of method and aim is legitimate only if it can be empirically certified by finding empirical correlations that show the method to be effective. But he construes this test of method in a way that rules out truth from the start, on the grounds that we cannot observe whether a theory is true, and so cannot directly check for a correlation between any method and that aim. Truth is in this way disqualified before the race has begun.

Peter Lipton is in the Department of History and Philosophy of Science, University of Cambridge, Free School Lane, Cambridge CB2 3RH, UK. 